\title{
HVPE growth and characterization of GaP on different substrates and patterned templates for frequency conversion devices
}

\section{Tassev}

vladimir.tassev@hanscom.af.mil

\section{Bliss}

\section{Snure \\ G. Bryant \\ R. Peterson \\ R. Bedford \\ C. Yapp \\ W. Goodhue \\ K. Termkoa}

Air Force Research Laboratory, Sensors Directorate, Hanscom AFB, MA 01731, USA

Air Force Research Laboratory, Sensors Directorate, Hanscom AFB, MA 01731, USA

Air Force Research Laboratory, Sensors Directorate, Hanscom AFB, MA 01731, USA

Air Force Research Laboratory, Sensors Directorate, Hanscom AFB, MA 01731, USA

Air Force Research Laboratory, Sensors Directorate, Wright Patterson AFB, OH 45433, USA

Air Force Research Laboratory, Sensors Directorate, Wright Patterson AFB, OH 45433, USA

Solid State Scientific Corporation, Hollis NH 03049, USA

University of Massachusetts, Photonic Center, Lowell, MA 01854, USA

University of Massachusetts, Photonic Center, Lowell, MA 01854, USA

This article describes efforts to achieve fast deposition of thick Quasi-Phase-Matched (QPM) GaP structures with high surface and structural quality on oriented patterned (OP) templates in a Hydride Vapor Phase Epitaxial (HVPE) process. These QPM structures will be incorporated in devices for conversion of frequencies from the near infrared to the mid infrared and THz regions, where powerful and tunable sources are in great demand for both military and civilian applications. In contrast with GaAs - the most studied OP QPM material - the two-photon absorption of GaP is predicted to be extremely low, which allows pumping with a number of convenient sources between $1-1.7 \mu \mathrm{m}$. Unpatterned GaP layers up to $370 \mu \mathrm{m}$ thick were grown with growth rates up to $93 \mu \mathrm{m} / \mathrm{hr}$ with high reproducibility on bare substrates. The layers demonstrated smooth surface morphology with RMS $1 \mathrm{~nm}$ and high structural quality with FWHM equal to 39 arcsec for layers grown on GaP and 112 arcsec for those grown on GaAs. Growth on OP-GaP templates resulted in $142 \mu \mathrm{m}$ thick QPM structures deposited at a growth rate of $71 \mu \mathrm{m} / \mathrm{h}$ with good vertical (normal to the layer surface) propagation of the initial pattern. When the growth was performed on OP-GaAs one of the domains showed a trend toward a faceting growth. Further investigations are in progress to equalize the vertical and lateral growth of the two domains, and determine the best orientation of the substrate and pattern in order to achieve structures thick enough for high power nonlinear applications. [DOI: 10.2971/jeos.2011.11017]

Keywords: hydride vapor phase epitaxy, nonlinear optical materials, quasi-phase matching, frequency conversion devices

\section{INTRODUCTION}

Development of direct coherent sources operating in the mid IR and $\mathrm{THz}$ regions started with the investigation of a great variety of binary and ternary semiconductor materials [1]-[4]. Resulting devices suffered from one or more common disadvantages: large size/weight, limited/undesirable wavelengths, lack of tunability, low efficiency, or the need for cumbersome cooling. To provide more options, the research included frequency conversion devices, initially based on bulk birefringent phase-matching (BPM) crystals [5]. Problems like thermal lensing, damage, and the large walk-off angle between the Poynting vector and the wave vector in BPM devices led to a search for alternative ways to keep the phase difference between the mixing waves constant, and ultimately to quasi-phase-matching (QPM). The first practical realization of QPM structures was in periodically-poled
$\mathrm{LiNO}_{3}$, but strong intrinsic absorption in this and other ferroelectric crystals limits their use to wavelengths shorter than $4 \mu \mathrm{m}$ [6]. In non-ferroelectric materials the QPM can be achieved by spatially inverting the nonlinear susceptibility, initially this was made by alternating wafers in a stack [7]. Optical losses at the interfaces and inability to achieve narrower domains needed for mid infrared generation, however, led to the development of more advanced techniques, such as OP thin templates.

More recently, OP-GaAs has emerged as a promising QPM material, due to its broad IR transparency, high nonlinear optical susceptibility, and ability to be grown with the necessary periodic structure [8]. Thus, QPM GaAs devices, nearly a millimeter-thick, with high conversion efficiency and out- 
put power, have been produced $[9,10]$. Such devices cannot take advantage of mature $\mathrm{Nd}, \mathrm{Yb}$, and Er-based pump lasers, however, due to significant two-photon absorption in the 1$1.7 \mu \mathrm{m}$ region [11].

This limitation can be overcome by using another semiconductor, GaP. Its two-photon absorption in the near-IR is negligible [12]. In addition, GaP has a relatively high nonlinear coefficient [13], higher thermal conductivity, lower thermal expansion and lower index of refraction [14] than GaAs, which make it a promising nonlinear material. GaP is an indirect band-gap semiconductor, with a broad transparency starting in the visible wavelengths, facilitating alignment in an optical setup $[15,16]$. Interest in QPM GaP has increased rapidly, and frequency conversion devices based on stacks of wafers with alternating inversion of the crystal orientation have been designed [17]. Growth of $\mathrm{GaP}$ on patterned templates has been initiated, using MBE [18, 19] and MOCVD [20] in an attempt to repeat the success of these techniques in fabricating QPM GaAs [21]. HVPE growth of QPM GaP is the only approach capable of producing the hundreds of $\mu \mathrm{m}$ thick apertures needed for high power applications. However, it is still in an initial stage [22] due to the reported low growth rates [23] or low crystal quality [24], although a great number of chemical paths, substrates, and modifications of the process have been tested. On the other side, the additional absorption band between 2 and $4 \mu \mathrm{m}$, persistently observed in all n-type GaP samples [25, 26] required an improvement of the control of impurity incorporation during the growth.

In this study we present an improved HVPE process for growing: (1) GaP layers on unpatterned $\mathrm{GaP}$ and GaAs substrates, and (2) GaP QPM structures on OP- GaAs and GaP templates. Two different techniques are used to produce the OP templates - wafer-fused bonding for preparation of OP$\mathrm{GaP}$ and sub-lattice MBE inversion for OP-GaAs. Some details related to the experimental procedure are given in Section 2. Section 3 is entirely dedicated to studying the growth process and establishing the optimal conditions for growth on unpatterned substrates (Section 3.1), half-patterned (stripes masked off) (Section 3.2) and fully- OP templates (Sections 3.3 and 3.4). An initial idea about the proper orientation of the template and the pattern is given at the end of this work.

\section{EXPERIMENTAL}

Growth experiments were performed in a horizontal lowpressure, hot-wall, 3-inch quartz reactor over the growth temperature range of $700-740{ }^{\circ} \mathrm{C}$, at supersaturation levels between 0.5 and 1.0, under pressure less than 10 Torr and a total mass flow less than $250 \mathrm{sccm}$. The reactor was supplied with a three-zone resistive furnace with precise control of the temperature. The inlet and the outlet gas flows were also computer controlled. $\mathrm{PH}_{3}, \mathrm{Ga}$, and $\mathrm{HCl}$, used as precursors, were delivered to the reactor volume by high purity $\mathrm{H}_{2}$. Unpatterned (100) GaP and (001) GaAs wafers with and without $4^{\circ}$ miscut towards (111) were used to grow $\mathrm{GaP}$ with initial phosphine or arsine preflow in order to study the utility of formation of an intermediate buffer layer. Surface and cross sections were characterized using Nomarski and Atomic Force Microscopy (AFM). The structural quality was determined by high resolution x-ray diffraction (HRXRD). The electrical properties were evaluated by room temperature Hall measurements using Van der Pauw method. The optical properties, transmission and absorption, were measured using a Cary 5000 spectrophotometer running from $400-2600 \mathrm{~nm}$ with a $2 \mathrm{~nm}$ fixed spectral bandwidth and a FT-IR scan from 2.5 to $15 \mathrm{~mm}$ with a bandwidth of $10 \mathrm{~nm}$. All characterizations were used as a feedback to optimize the conditions of growth. The growths were conducted further on halfpatterned (stripes masked off) GaP templates to study the behavior of the opposite oriented domains separately at different orientations towards the major flat of the wafers. Eventually experiments on fully-patterned sub-lattice MBE OP-GaAs wafer-fused bonding and OP-GaP templates were performed. In difference to the former approach the waferfused bonding is an innovative simpler procedure, which does not require using MBE in the process of wafer preparation. The process consists in the next simple steps. First, two (100) GaP wafers with inverted crystallographic orientations are aligned and fused at high temperature. After bonding, the top $\mathrm{GaP}$ substrate is removed to within $4-5 \mu \mathrm{m}$ using mechanical polishing and chemical wet etching. The sample is then patterned via standard photolithography, followed by a bromine ion beam assisted dry etching to remove completely the top $\mathrm{GaP}$ layer, which was not covered with photoresist. The final step to create the grating domains is to remove the photoresist.

Further details related to the growth experiment are given in [22].

\section{RESULTS AND DISCUSSION}

\subsection{Growth on unpatterned GaP and GaAs substrates}

Growth on bare GaP substrate was investigated in two onehour-long experiments as reactor pressure and the temperatures of the gallium boat and of the mixing zone were kept constant. The temperature of the substrate was gradually increased from $707^{\circ} \mathrm{C}$ to $725^{\circ} \mathrm{C}$ at constant supersaturation of about 0.5 . Once the optimal for this serial of experiments growth rate, i.e. the highest one with the lowest surface roughness, was determined the supersaturation was gradually changed from about 0.5 to about 0.8 at the optimal growth temperature. The average roughness as a factor of the surface quality was monitored in conjunction with the growth rate and supersaturation.

The results indicated that the substrate temperature has a strong influence on both growth rate and surface morphology. Like the findings of other authors [27], the growth rate increases with increasing temperature (Figure 1a) but at $720^{\circ} \mathrm{C}$ 

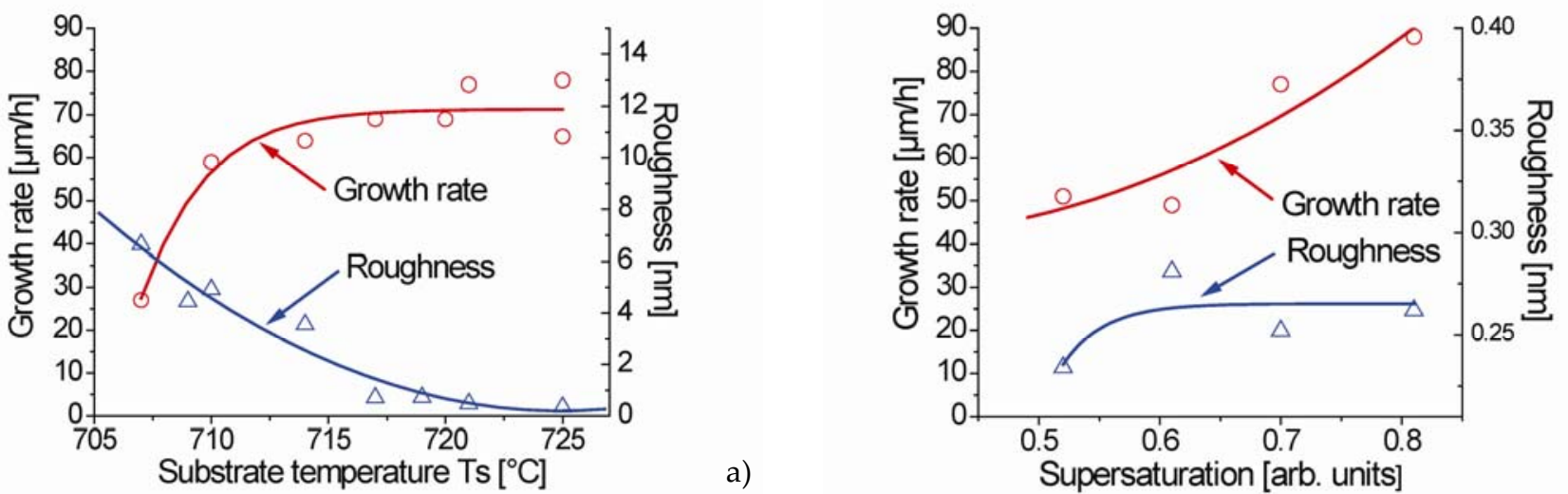

FIG. 1 Growth rate and layer roughness as a function of substrate temperature (a) and supersaturation (b) during growth on bare GaP substrate.
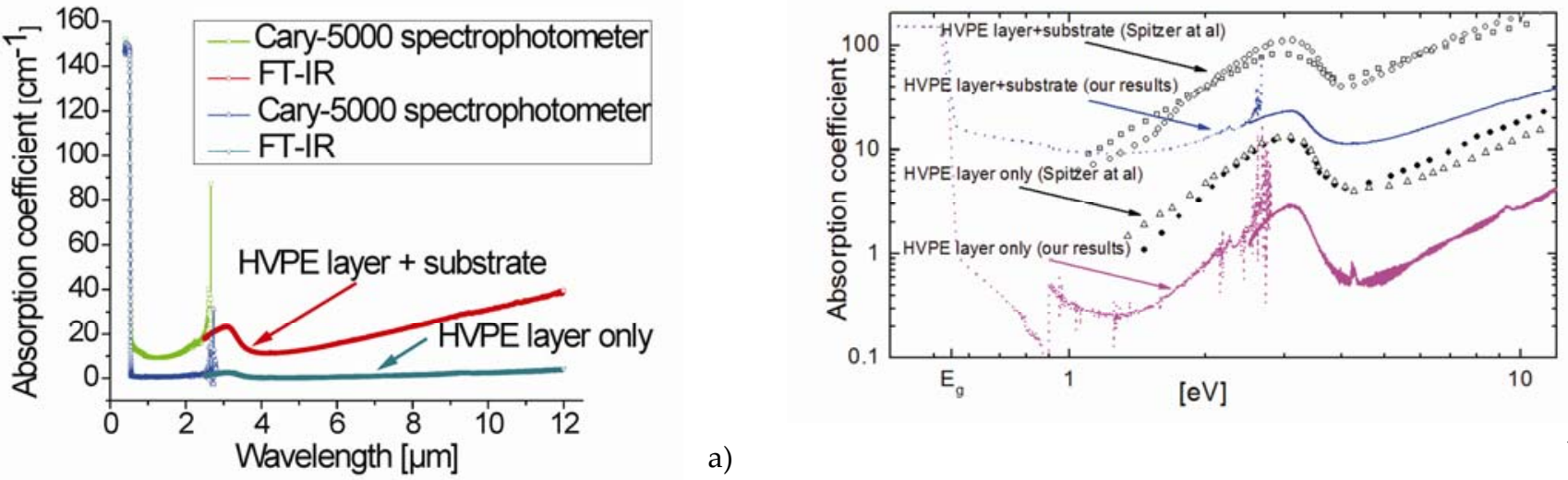

a)

FIG. 2 Combined absorption spectra of a HVPE grown unpatterned GaP layer with and without the substrate (colored lines) plotted in linear (a) and logarithmic (b) scale compared with related data (the black symbols) obtained by previous authors [25] (b).

it levels off at $70 \mu \mathrm{m} / \mathrm{h}$. The temperature dependence of the growth rate indicates that the growth is kinetically rather than thermodynamically limited similar to other findings [28, 29]. The difference between these 2 regimes is that while in a kinetically limited process events at a molecular level like removal of the chemisorbed chlorine as $\mathrm{HCl}$ play ratelimiting role [30], in a mass limited process factors like mass flow rate and, actually, what portion of the flow reacts with the substrate [29] will have more pronounced impact than the temperature. Similar conclusion, that HVPE (in case of GaAs) is mainly governed by surface kinetics, can be also found in [31]. The surface morphology also improves with increasing temperature. For example, at higher growth temperatures the wave texture [27], which is typical for most of the III-V semiconductors, disappears as the roughness of the grown layer becomes as low as the roughness of the initial substrate. This makes it possible to perform further regrowths and build additional layer thickness in more than one experiment without polishing the layer surface. Similarly, small changes in the supersaturation can result in strong changes in the growth rate, as the dependence is proportional, i.e. an increase in supersaturation leads to an increase in the growth rate (Figure 1b). It seems, however, that in the studied range supersaturation has small influence on the surface morphology. Although some finer structural effects like formation of particles in the stream that could rest on the layer surface (thermophoresis) can initiate defects and thus deserve more attention.

Avoiding the additional absorption observed between 2 and $4 \mu \mathrm{m}$ was one of the major tasks of this study. Figure $2 \mathrm{a}$ and Figure $2 \mathrm{~b}$ show the combined (Cary 5000 and FT-IR) absorption spectra (the colored lines) of two unpatterned GaP samples. One of the samples has a thickness of $685 \mathrm{~mm}$ and includes the substrate and the HVPE-grown layer while the other one is $370 \mathrm{~mm}$ thick and represents only the HVPE regrowth after the substrate was polished off. Figure 2a shows in a linear scale that the HVPE layer has much lower absorption in comparison to the case when the substrate and the HVPE layer were still together. The same data was plotted in a logarithmic scale on Figure $2 b$ and compared with related data obtained by other authors [25, 26]. The comparison shows that our HVPE material has lower absorption, which indicates a lower impurity concentration.

The combined absorption spectra in Figure $2 \mathrm{~b}$ exhibit a small jump in the signal at $0.8 \mu \mathrm{m}$, which is an instrumental artifact as a result of the change of the detector, as well as the noise at 2.4-2.6 $\mu \mathrm{m}$ (Figure 2a and Figure 2b), which is due to a signal-to-noise ratio issue related to the detector/source 


\begin{tabular}{|c|c|c|c|}
\hline $\begin{array}{ll}\text { Characteristic } \downarrow & \text { Substrate } \rightarrow\end{array}$ & $\mathrm{GaP}$ on $\mathrm{GaP}$ with $\mathrm{PH}_{3}$ preflow & $\begin{array}{l}\mathrm{GaP} \text { on } \mathrm{GaAs} \text { with } \mathrm{PH}_{3} \text { pre- } \\
\text { flow }\end{array}$ & $\begin{array}{c}\mathrm{GaP} \text { on } \mathrm{GaAs} \text { with } \mathrm{AsH}_{3} \\
\text { preflow }\end{array}$ \\
\hline Growth rate $[\mu \mathrm{m} / \mathrm{h}]$ & 52.5 & 54 & 65 \\
\hline XRD FWHM [arc-sec] & 39 & 112 & 189 \\
\hline RMS [nm] $(20 \mu \mathrm{m}$ spot $)$ & 0.725 & 0.667 & 0.637 \\
\hline Carrier concentration & $n=7.4 .10^{16}$ & $n=5.0 .10^{16}$ & $n=1.8 .10^{16}$ \\
\hline Mobility $\left[\mathrm{cm}^{2} \mathrm{~V}^{-1} \mathrm{sec}^{-1}\right]$ & 126 & 119 & 94 \\
\hline
\end{tabular}

TABLE 1 Growth of GaP on bare GaP and GaAs substrates in $\mathrm{PH}_{3}$ or $\mathrm{AsH}_{3}$ preflows.

combination. The gradual rise/peak in the FTIR data for the HVPE sample around $4.1 \mu \mathrm{m}$ is, probably, due to a direct transition from a shallow donor to the upper conduction band or to a conduction band-to-band transition [32, 33].

Table 1 lists the results from three two-hour growths of $\mathrm{GaP}$ on semi-insulated (SI) unpatterned $\mathrm{GaP}, \mathrm{GaAs}$ under $\mathrm{PH}_{3}$ preflow and $\mathrm{GaAs}$ under $\mathrm{AsH}_{3}$ preflow. The preflows were initiated at $400{ }^{\circ} \mathrm{C}$ and aimed to prevent the thermal decomposition of the substrate. Another goal was to deposit an intermediate GaAsP buffer layer to make the transition between these two materials smoother.

One can see that growth rates increase from column 1 to columns 2 and 3 at the expense of the structural quality, due to the lattice and thermal mismatch between the GaP layer and the GaAs substrate. It also shows the utility of formation of an intermediate layer, which has a favorable influence on the final surface quality, slightly reducing the surface roughness. The changes in the carrier concentration and mobility need a deeper understanding of the mechanism of formation of point defects in the intermediate layer [34].

Starting with the study of the behavior of different reactor components, the optimization of the growth conditions in this serial of experiments continued with their testing in 4and 8-hour long experiments. The reactor operated equally and after the optimization all experiments were conducted without any visible parasitic reactions in front or around the substrates. The 4 -hour growth experiments were marked by their high reproducibility of the layer characteristics and the high layer quality. Etch pits and small hillocks that slightly deteriorated the surface morphology appeared after 8 hours of growth, but even then almost no additional absorption band between 2 and $4 \mu \mathrm{m}$ was observed in the sample although it existed in the used substrate. The growth conditions, initially optimized for growth on unpatterned $\mathrm{GaP}$ substrates, were further applied in longer experiments for growth on unpatterned GaAs and on different GaP and GaAs OP templates, when in some cases the growth was even faster. Figure 3 shows the optimized growth rates achieved as a function of duration of growth.

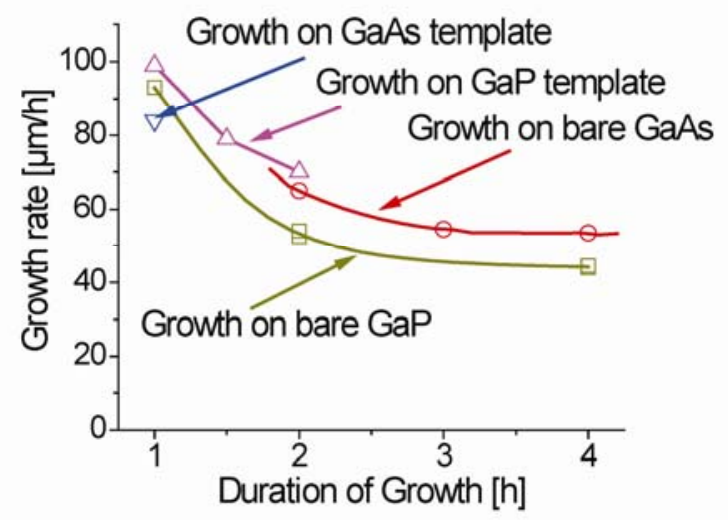

FIG. 3 Growth rate as a function of duration of growths performed on bare and on patterned GaP and GaAs substrates.

In all cases the growth rate reduces with time but after four hours it levels off to a rate which for growth on GaP substrates is about twice as high as the reported levels [23] for material with relatively good quality. In the HVPE process the growth rate is controlled by:

(1) the reduction of parasitic deposits in the process;

(2) obtaining the appropriate balance between absorption of $\mathrm{Ga}(\mathrm{GaCl})$ and $\mathrm{P}\left(\mathrm{PH}_{3}\right)$ precursors and desorption of $\mathrm{HCl}$ thereby, reducing competition between $\mathrm{P}$ and $\mathrm{Cl}$ for available surface sites [29, 30];

(3) the appropriate balance between $\mathrm{PH}_{3}$, and the generated during the pyrolysis $\mathrm{P}_{2}^{+}$and $\mathrm{P}_{4}^{2+}[28]$.

$\mathrm{PH}_{3}$ has a higher equilibrium constant in the reaction with $\mathrm{GaCl}$ in comparison to the other two species and plays the main role in desorption of chlorine from the layer surface. By decomposing on the growing surface, $\mathrm{PH}_{3}$ supplies the chlorine which is already chemisorbed in the layer with atomic hydrogen more rapidly than the supply that could possibly come from dissociation of molecular hydrogen [30]. This increase of the growth rate at uncompleted $\mathrm{PH}_{3}$ pyrolysis can be, however, at the expense of the surface morphology. This is because the atomic phosphorus, released at the $\mathrm{PH}_{3}$ pyrolysis, can form molecular $\mathrm{P}_{2}$, whose probability to react with Ga is now much smaller than for atomic phosphorus. Thus, the un-reacted gallium can condense as droplets on the layer surface. Further, the Ga-droplets can initiate forming of hill- 


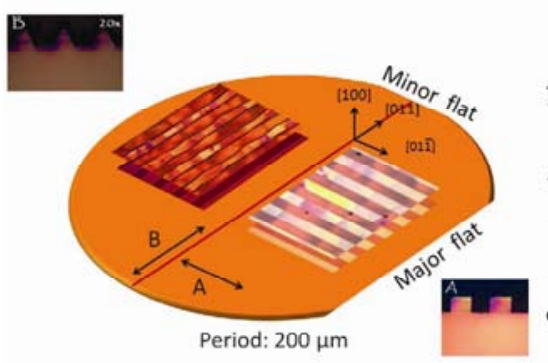

a)

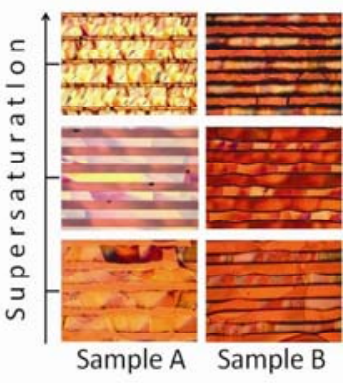

b)
FIG. 4 Growth of GaP on half-patterned (100) GaP templates (a) at different supersaturation (b).

ocks via vapor-liquid-solid growth [30]. The choice of appropriate combination of the process parameters like substrate and Ga temperature, reactor pressure $[35,36]$, and supersaturation facilitated the above mentioned mechanisms and allowed to achieve growth rates as high as $93 \mu \mathrm{m} / \mathrm{h}$ on unpatterned templates.

\subsection{Growth on half-patterned GaP substrates}

Selective area growth of GaP has been performed before [20]. We studied the growth on half-patterned, i.e. stripes masked off, templates in order to investigate the behavior of the two domains with opposite crystallographic orientations separately. For this purpose one (100) GaP wafer was cut in half parallel to its major flat (the major and minor flats are cuts used in the wafer industry to denote specific crystallographic orientations). Two patterns, designated Pattern A and Pattern B, with inverted crystallographic orientations were prepared in mutually perpendicular directions, using a $100 \mu \mathrm{m}$ wide grating mask, where the uncovered areas were prevented from growth by deposition of a $0.1 \mu \mathrm{m}$ thick silicon nitride layer. The stripes of pattern A were directed along [01i1] (perpendicular to the major flat), whereas those of pattern B were directed along [011] (perpendicular to the minor flat).

Figure 4a shows cross sections after one hour of growth on samples A and B. It turns out that under certain conditions the growth on $\mathrm{A}$ is vertical, while the growth on $\mathrm{B}$ shows a trend toward a facet growth. The growth rate on A was $34 \mu \mathrm{m} / \mathrm{h}$, whereas B grew slightly slower-at $30 \mu \mathrm{m} / \mathrm{h}$. These cross section images are of samples grown at a moderate level of supersaturation. They are shown as the middle line of images in Figure $4 \mathrm{~b}$ and represent optimal supersaturation.

Figure $4 \mathrm{~b}$ also shows the top surfaces of $\mathrm{A}$ and $\mathrm{B}$ grown at lower (bottom line of figures) and higher (upper line of figures) supersaturation. One can see that any deviation in the supersaturation from the optimal level dramatically changes the width of the growing domains. It also turns out that orientation $A$, which has better performance at the optimal supersaturation, is actually more sensitive to changes in the supersaturation.

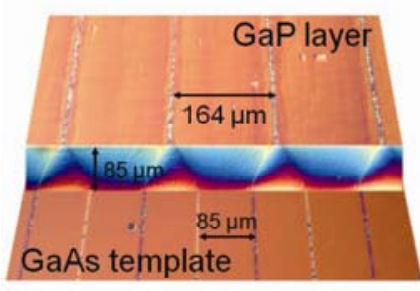

a)

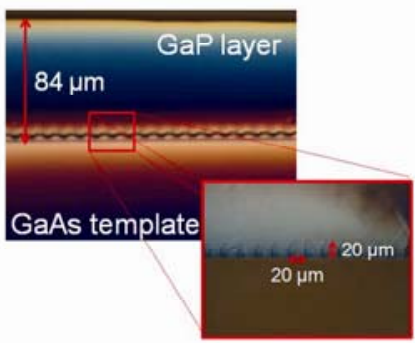

b)
FIG 5 Growth of GaP on OP MBE GaAs templates with grating periodicities equal to $170 \mu \mathrm{m}(\mathrm{a})$ and $20 \mu \mathrm{m}$ (b).

\subsection{Growth on sub-lattice MBE inversion OP GaAs templates}

The first attempt to grow GaP on fully OP substrates was performed on MBE inversion OP-GaAs templates. The inversion of the crystallographic orientation and hence the sign of the nonlinear susceptibility of the patterns, produced at Stanford University, was achieved by deposition of a thin Ge intermediate layer in a MBE process. Two different patterns, with grating periodicities equal to 170 and $20 \mu \mathrm{m}$, respectively, were tested over a one hour growth time.

The growth rate in both areas was $85 \mu \mathrm{m} / \mathrm{h}$. Figure 5a (a 3D reconstruction of images of the cross section and the surface before and after growth) shows that in the case of the $170 \mu \mathrm{m}$ wide grating one of the domains grows at the expense of the other domain. Thus, after one hour of growth this domain is already $164 \mu \mathrm{m}$ wide, while the width of the other one is only $6 \mu \mathrm{m}$. In the case of the $20 \mu \mathrm{m}$ wide grating, Figure 5b, after $20 \mu \mathrm{m}$ of growth the lateral growth prevails and the pattern disappears. Due to the lattice and thermal mismatch between the layer and the template, the growth of $\mathrm{GaP}$ on $\mathrm{GaAs}$ patterns is less favorable. These initial experiments, however, proved the concept that $\mathrm{GaP}$ can follow patterns even on foreign substrates.

\subsection{Growth on wafer fusion bonded OP gap templates}

The wafer fusion bonding technique for the preparation of OP-GaP templates (WF/OP-GaP), in contrast to the sublattice MBE inversion approach (which in case of GaP uses Si intermediate layer to reverse the polarity), does not require expensive equipment and is a relatively simple but effective procedure. It consists of several steps involving bonding of 2 wafers with opposite crystallographic orientations at high temperature and a consequent pattern gratings using photolithography. The process, developed first for GaAs [37, 38], was recently adopted for $\mathrm{GaP}$ [39]. More details about preparation of the templates will be published elsewhere [40].

The deposition of GaP was performed under optimized growth conditions on such WF/OP-GaP templates. Each tem- 


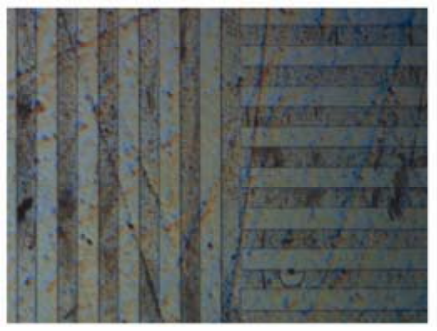

a)

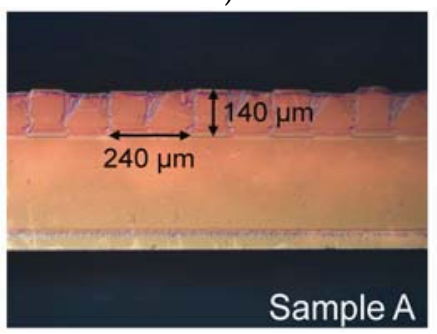

c)

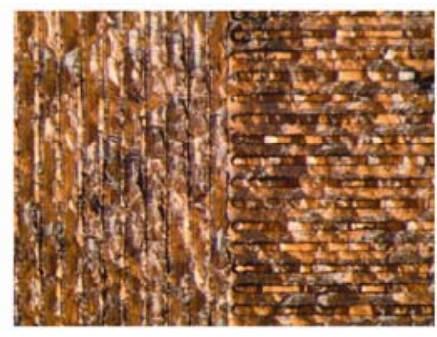

b)

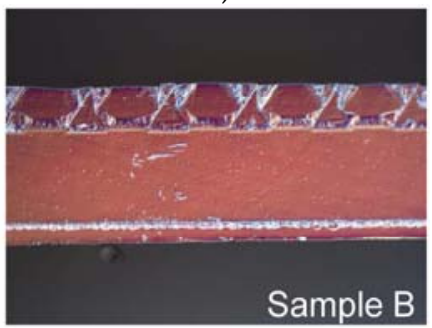

d
FIG 6 Growth on wafer fusion bonded OP-GaP templates: the wafer before (a) and after (b) growth as the horizontal pattern is with orientation along [01 ], while the vertical one is along 011] (b). (c) (sample A) and (d) (sample B) represent their cross sections.

plate possessed two patterns with a periodicity of about $240 \mu \mathrm{m}$ in two mutually perpendicular directions - the same as the directions of the patterns on half-patterned templates (see Section 3.2). The duration of growth was two hours at an average growth rate of $71 \mu \mathrm{m} / \mathrm{h}$. A $142 \mu \mathrm{m}$ thick QPM GaP structure was achieved.

Like the growth on half-patterned templates, the growth on the fully-patterned wafer bonded templates (Figure 6a - before growth) was better when the stripes of the pattern were directed along [01ī] (perpendicular to the major flat) - sample A (Figure 6b - the horizontal pattern, and Figure 6c - cross section), than when they were directed along [011] (perpendicular to the minor flat)-sample B (Figure $6 \mathrm{~b}$ - the vertical pattern, and Figure $6 \mathrm{~d}$ - cross section). Similar results - that growth in different orientations on unpatterned and patterned substrates leads to different growth rates and different mesa shapes - have been reported for some related materials, such as GaAs [31] and InP [41]. The difference in the deposition rates when growth was conducted in different orientations can be attributed to the different surface energy. The shape of the mesas results from the hierarchy of the growth rates of the low index faces. A qualitative on-atomiclevel explanation suggests that the chemisorbed on the surface $\mathrm{GaCl}$ needs some time to react with the available atomic hydrogen and return as $\mathrm{HCl}$ to the vapor phase, allowing phosphorus atoms to absorb on the substrate. This time seems to be proportional to the number of bonds that $\mathrm{GaCl}$ makes with the substrate. This number is different for the different orientations. Thus the growth can be facilitated or hindered and this will influence on the mesa shape, too. Further investigations, which aim to confine the lateral growth, to equalize the growth rates and the quality of the two oppositely oriented domains, and thus to achieve at least $500 \mu \mathrm{m}$ thicknesses as needed for high power nonlinear applications, are currently in progress.

\section{CONCLUSION}

HVPE growth of GaP was performed on different types of unpatterned substrates and patterned templates. A number of useful growth dependencies, related to the growth rate and different layer characteristics, were established. Determining the optimal for the studied range growth conditions and establishing a process for fast deposition allowed to achieve a maximal growth rate of $93 \mu \mathrm{m} / \mathrm{hr}$, and to grow $\mathrm{GaP}$ layers up to $370 \mu \mathrm{m}$ thick on unpatterned GaP template. The layers possess a smooth surface morphology (AFM) with an average roughness less than $1 \mathrm{~nm}$, and high structural quality (XRD), with FWHM = 39 arc-sec for growth on GaP and FWHM $=112$ arc-sec for growth on GaAs. A new wafer fusion bonding process for fabricating orientation-patterned $\mathrm{GaP}$ templates was developed and tested for its feasibility to provide a medium for the growth of hundreds of microns thick QPM structures. A good quality GaP QPM structure, $142 \mu \mathrm{m}$ thick, was successfully grown, with an average growth rate of $71 \mu \mathrm{m} / \mathrm{h}$ on such templates, following the initial pattern. The optimal orientation of the pattern towards to the major flat of the wafer was determined as well.

\section{ACKNOWLEDGEMENTS}

The authors thank to the Air Force Office for Scientific Research for their financial support, and also Ms. Sandra Wentzell for preparing the half-patterned templates.

\section{References}

[1] A. Codard, "Infrared $(2-12 \mu \mathrm{m})$ solid-state laser sources: a review" CR Phys. 8, 1100-1128 (2007).

[2] A. Zajac, M. Skorczakowski, J. Swiderski, and P. Nyga, "Electrooptically Q-switched mid-infrared Er: YAG laser for medical applications" Opt. Express 12, 5125-5130 (2004).

[3] J. G. Kim, L. Shterengas, R. U. Martinelli, and G. L. Belenky, "High -power room-temperature continuous wave operation of 2.7 and $2.8 \mu \mathrm{m} \mathrm{In(Al)GaAsSb/GaSb} \mathrm{diode} \mathrm{lasers"} \mathrm{Appl.} \mathrm{Phys.} \mathrm{Lett.} \mathrm{83,}$ 1926-1928 (2003).

[4] R. Kaspi, A. P. Ongstad, C. C. Dente, J. R. Chavez, M. L. Tilton, and D. Cianardi, "High power and high brightness from an optically pumped InAs/InGaSb type-II midinfrared laser with low confinement" Appl. Phys. Lett. 81, 406-408 (2002).

[5] S. B. Blickenstaff, A. M. Sarangan, T. R. Nelson Jr., K. D. Leedy, and D. L. Agresta, "Influence of shadow mask design and deposition methods on non-planar dielectric material deposition" J. Microlithogr., Microfab. and Microsyst. 4, 0230151-0230156 (2005).

[6] L. E. Myers, W. R. Bosenberg, "Periodically poled lithium niobate and quasi-phase-matched optical parametric oscillators", J. Quant. Elect. 33, 1663-1672 (1997). 
[7] A. Szilagyi, A. Hordvik, and H. Schlossberg, "A quasi-phasematching technique for efficient optical mixing and frequency doubling"' J. Appl. Phys. 47, 2025-2032 (1976).

[8] L. A. Eyres, P. J. Tourreau, T. J. Pinguet, C.B. Ebert, J.S. Harris, M.M. Fejer, L. Becouarn, B. Gerard, and E. Lallier, "All-epitaxial fabrication of thick, orientation-patterned GaAs films for nonlinear optical frequency conversion" Appl. Phys. Lett. 79, 904-906 (2001).

[9] C. Lynch, D. Bliss, T. Zens, V. Tassev, P. Kuo, A. Lin, J. Harris, M. Fejer, and P. Schunemann, Mm-thick orientation-patterned GaAs for IR and THz generation (ICVGE-13, Salt Lake City, 12-17 August 2007).

[10] C. Kieleck, M. Eichhorn, A. Hirth, D. Faye, and E. Lallier, "Highefficiency $20-50 \mathrm{kHz}$ Mid-infrared orientation-patterned GaAs optical parametric oscillator pumped by a $2 \mu \mathrm{m}$ holmium laser" Opt. Lett. 34, 262-264 (2009).

[11] W. Hurlbut, Y. Lee, K. Vodopyanov, P. Kuo, and M. Fejer, "Multiphoton absorption and nonlinear refraction of GaAs in the midinfrared" Opt. Lett. 32, 668-670 (2007).

[12] L. H. Bechtel and W. L. Smith, "Two-photon absorption in semiconductors with picoseconds laser pulses" Phys. Rev. B 13, 3515 -3522 (1976).

[13] I. Shoji, T. Kondo, A. Kitamoto, M. Shirane, and R. Ito, "Absolute scale of second-order nonlinear-optical coefficients" J. Opt. Soc. Am. B 14, 2268-2294 (1997).

[14] M. E. Thomas, D. W. Blodgett, D. V. Hahn, and S. G. Kaplan, "Characterization and modeling of the infrared properties of GaP and GaAs" Proc. SPIE 5078,159-168 (2003).

[15] I. Shoji, and T. Taira, "Transmittance spectra of GaAs and GaP in far-infrared region" Ultraviolet Synchrotron Orbital Radiation Activity Report 2001,142-143 (2002).

[16] A. N. Pikhtin, and D. A. Yaskov, "Infrared absorption in gallium phosphide" Phys. Status Solidi (b) 34, 815-824 (1969).

[17] I. Tomita, "Fabrication and characterization of a quasi-phasematched GaP optical device for terahertz-wave generation" Opt. Mater. 32, 323-328 (2009).

[18] T. Matsushita, I. Ohta, and T. Kondo, "Quasi-phase-matched parametric fluorescence in a periodically inverted GaP waveguide" Appl. Phys. Exp. 2, 0611011-0611013 (2009).

[19] T. Matsushita, T. Yamamoto, and T. Kondo, "Epitaxial growth on spatially inverted GaP for quasi-phase-matched nonlinear optical devices" Jap. J. Appl. Phys. 17, L408-410 (2007).

[20] J. W. Lee, J. Salzman, D. Emerson, J. Shealy, and J. M. Ballantyne, "Selective area growth of GaP on Si by MOCVD" J. Cryst. Gr. 172, 53-57 (1997).

[21] C. Lynch, V. Tassev, D. Bliss, and C. Bryant, Growth on orientation patterned semiconductors for nonlinear optical frequency conversion (Advances in Optical Materials, San Jose, 11 0ct. 2009).

[22] V. Tassev, D. Bliss, C. Lynch, C. Yapp, W. Goodhue, and K. Termkoa, "Low pressure-temperature-gas flow HVPE growth of GaP for nonlinear optical frequency conversion devices" J. Cryst. Growth 312, 1146-1149 (2010).

[23] C. C. Wang, and S. H. McFarlane, "Epitaxial growth and characterization of GaP on insulating substrates" J. Cryst. Growth $13 / 14,262-267$ (1972).
[24] W. G. Oldham, "Vapor growth of GaP on GaAs substrates" J. Appl. Phys. 36, 2887-2890 (1965).

[25] W. G. Spitzer, M. Gershenzon, C. J. Frosch, and D. F. Gibbs, "Optical absorption in n-type gallium phosphide" J. Phys. Chem. Sol. 11 3/4, 339-341 (1959).

[26] D. A. Yasakov, A. N. Pikhtin, and V. I. Ulyanov, "Optical properties of gallium phosphide grown by floating zone" Mat. Res. Bull. 4, 839-848 (1969).

[27] N. J. Kadhim, and D. Mikherjee, "Growth defects associated with MBE deposited GaAs layers" Vacuum 55, 249-253 (1999).

[28] M. Weyers, and M. Sato, "Growth of GaP by MOVPE at very low pressure: kinetics and carbon incorporation" J. Cryst. Growth 115, 469-473 (1991).

[29] D. W. Shaw, "Kinetic aspects in the vapor phase epitaxy of III-V compounds" J. Cryst. Growth 31, 130-141 (1975).

[30] R. Karlicek, D. Mitcham, J. Ginocchio, and B. Hammarlund, "The effect of formation on the morphology and growth rate of InP grown by hydride vapor phase epitaxy" J. Electrochem. Soc. 134, 470-474 (1987).

[31] E. Lafon, J. Napierala, D. Castellici, A. Pimpinelli, R. Cadoret, and B. Gerald, "Selective growth of GaAs by HVPE: keys for accurate control of the growth morphologies" J. Cryst. Growth 222, 482-496 (2001).

[32] J. W. Allen, and J. W. Hodby, "Infrared absorption in GaP-GaAs Allows: Absorption in n-type materials" Proc. Phys. Soc. 82, 315323 (1963).

[33] J. W. Hodby, "Infrared absorption in GaP-GaAs Alloys: Absorption in p-type materials" Proc. Phys. Soc. 82, 324-326 (1963).

[34] D. T. J. Hurle, "Charged native points defects in GaAs and other III-V compounds" J. Cryst. Growth 237, 1621-1627 (2002).

[35] K. Gruter, M. Deschler, H. Jurgensen, R. Beccard, and P. Balk, "Deposition of high quality GaAs films at fast rates in the LP-CVD system" J. Cryst. Growth 94, 607-612 (1989).

[36] E. Putz, E. Veuhoff, K. H. Bachem, P. Balk, and H. Luth, "Low pressure vapor phase epitaxy of GaAs in a halogen transport system" J. Electrochem. Soc. 128, 2202-2206 (1981).

[37] C. Kieleck, M. Eichhorn, A. Hirth, D. Faye, and E. Lallier, "20-50 kHz "Mid-Infrared OP-GaAs OPO”, Proc. CLEO/QELS (2008).

[38]. J. Li, D. Fenner, K. Termkoa, M. Allen, P. Moulton, C. Lynch, D. Bliss, and W. Goodhue, "Wafer-fused orientation pattern GaAs" Proc. SPIE 6875, 68750H (2008)

[39] K. Termkoa , V. Mathur , X. Qian , W. Goodhue, D. Bliss, R. Peterson, and V. Tassev, Development of a Wafer Fusion Process for Producing Patterned GaP Templates (APS March Meeting, Pittsburgh, 16-20 March 2009).

[40] K. Termkoa, S. Vangala, W. Coodhue, R. Peterson, R. Bedford, V. Tassev, C. Lynch, and D. Bliss, "Orientation-patterned GaP using wafer fusion technique" Opt. Mater. (submitted).

[41] R. Karlisek, B. Segner, J. Wynn, A. Becker, U. Chakrabarti, and R. Logan, "Regrowth of semi-insulating InP around etched mesas using hydride vapor phase epitaxy" J. Electrochem. Soc. 137, 2639-2642 (1990). 